\title{
EFEITO DO PACLOBUTRAZOL NO CONTROLE DA DIFERENCIAÇÃO FLORAL NATURAL DO ABACAXIZEIRO CV. SMOOTH CAYENNE ${ }^{1}$
}

\author{
ANDRÉA MARIAANTUNES ${ }^{2}$, ELIZABETH ORIKA ONO $^{3}$, ALOÍSIO COSTA SAMPAIO $^{4}$
}

RESUMO- O abacaxizeiro é uma planta de grande importância econômica, porém seu florescimento natural causa sérios problemas, tornando seu manejo difícil devido à desuniformidade de frutos e colheitas, elevando o custo de produção. O objetivo deste trabalho foi manipular o florescimento do abacaxizeiro, contribuindo para uma produção uniforme colocada no mercado, nos meses de menor oferta. Utilizou-se o Paclobutrazol (PBZ) nas concentrações de 100; 150 e $200 \mathrm{mg} \mathrm{L}^{-1}$, em 2; 3 ou 4 aplicações via foliar, em plantas de abacaxi cv. Smooth Cayenne, no município de Presidente Alves-SP. O delineamento empregado foi em blocos ao acaso, com 10 tratamentos e três repetições, com 40 plantas por parcela experimental. No período de 100 a 150 dias após a primeira aplicação dos tratamentos, efetuaram-se as contagens de inflorescências presentes no centro da roseta foliar das plantas. Todos os tratamentos com Paclobutrazol inibiram a diferenciação floral natural do abacaxizeiro, recomendando-se a concentração de $150 \mathrm{mg} \mathrm{L}^{-1} \mathrm{em} \mathrm{duas}$ aplicações, com início em abril, a intervalo de 15 dias.

Termos para indexação: Ananas comosus var. comosus, florescimento, inibidor vegetal, crescimento vegetativo.

\section{EFFECT OF PACLOBUTRAZOL IN THE CONTROL OF THE NATURAL FLOWERING DIFFERENCE OF 'SMOOTH CAYENNE' PINEAPPLE PLANT}

\begin{abstract}
The pineapple plant is a plant of great economical importance, however, its natural flowering causes serious problems, turning it difficult to manage it due to the fruit and harvest irregularity, elevating the production's cost. The objective of this work was to manipulate the pineapple flowering contributing indeed for a more uniform production on the market, during the months of lower offer. The Paclobutrazol was used in pineapple plants cv. Smooth Cayenne at concentrations of 100; 150 and $200 \mathrm{mg} \mathrm{L}^{-1}$ and applied in 2, 3 or 4 times, in Presidente Alves-SP. The experimental design was randomized blocks with 10 treatments and three replications, each experimental portion of 40 plants. In the period of 100 to 150 days after the first application of the treatments, the number of inflorescences present in the center of the plants were counted each week. All of the treatments involving Paclobutrazol were able to inhibit pineapple natural floral differentiation. The product is recommended to be used at a concentration of $150 \mathrm{mg} \mathrm{L}^{-1}$, applied twice, with a fifteen-day interval between applications, beginning in April.
\end{abstract}

Index Terms: Ananas comosus var. comosus, flowering, growth inhibitor, vegetative growth.

\section{INTRODUÇÃO}

O abacaxizeiro (Ananas comosus (L.) Merrill), originário da América do Sul, encontra-se difundido nas regiões tropicais e subtropicais do mundo, onde predomina o plantio da cultivar Smooth Cayenne, devido à sua qualidade e aceitação comercial (Reinhardt \& Souza, 2000). A produção mundial de abacaxi em 2002 foi de aproximadamente 14.375 .000 toneladas, com a distribuição de $14 \%$ na Tailândia, de $12 \%$ nas Filipinas e $10 \%$ no Brasil (FAO, 2004). A produção do Brasil em 2004 (em mil frutos) foi de 1.420.298, e a do Estado de São Paulo, de 79.860 (IBGE, 2004). A região de Bauru é a segunda produtora de abacaxi do Estado, com área cultivada de 800 ha e, aproximadamente, oito milhões de mudas plantadas anualmente (Sampaio, 2003).

Análises de sazonalidade de preços nas principais Ceasas do País indicam que o período de escassez de oferta com elevação dos preços médios ocorre nos meses de fevereiro a abril. Nos meses de maio a outubro, os preços ficam em torno da média anual, enquanto, nos meses de novembro a janeiro, em virtude do excesso de oferta do produto, os preços ficam abaixo da média anual.

Isso acontece pelo manejo com uso do ethephon, o que permite a antecipação da diferenciação floral. Na ausência desse manejo, o processo de diferenciação floral natural ocorre nos meses de junho e julho, quando os dias são curtos, e as temperaturas são baixas no Estado de São Paulo. Segundo Barbosa et al. (1998), Rabie et al. (2000) e Cunha et al. (2003), o florescimento natural dessa bromeliácea causa sérios inconvenientes, como a desuniformidade de maturação dos frutos e período de colheitas, elevando assim o custo de produção.

O manejo mais utilizado para uniformizar o período de colheita do abacaxi é o emprego de reguladores vegetais que permitem a indução artificial da diferenciação floral. Entretanto, geram a concentração da safra nos meses de novembro a janeiro, quando os preços estão abaixo da média anual. Outra forma de

${ }^{1}$ (Trabalho 145-07). Recebido em: 06-06-2007. Aceito para publicação em: 18-02-2008.

Universidade Estadual Paulista - UNESP, Pós-Graduanda do Programa de Ciências Biológicas (Botânica), Dept ${ }^{\circ}$ de Botânica, IB, CP 510, CEP:18618000, Botucatu-SP. Tel. (14)3811-6053. E-mail: andreamantunes@yahoo.com.br. Bolsista CAPES.

${ }^{2}$ Universidade Estadual Paulista - UNESP, Prof ${ }^{a}$ Adjunta/Livre-Docente do Dept ${ }^{\circ}$ de Botânica, IB, CP 510, CEP:18618-000, Botucatu-SP. Tel. (14)38116053. E-mail: eoono@ibb.unesp.br.

${ }^{3}$ Universidade Estadual Paulista - UNESP, Prof. Assistente/Doutor do Dept ${ }^{\circ}$ de Ciências Biológicas/FC/Bauru-SP, CP 473, CEP 17033-360, Tel. (14) 3103-6078. E-mail: aloísio@fc.unesp.br. 
interferir no processo de diferenciação floral natural do abacaxizeiro e obter a produção uniforme e em época de melhores preços de mercado seria a utilização de inibidores vegetais (Min \& Bartholomew, 1996; Barbosa et al., 1998; Cunha et al., 2003). Entre os inibidores vegetais, o Paclobutrazol é um efetivo inibidor da síntese de etileno, devido ao efeito inibidor na conversão de ACC (1- aminociclopropano-1-carboxílico) a etileno (Tari \& Mihalik, 1998).

Assim, objetivando-se contribuir para facilitar o escalonamento da produção e comercialização de abacaxi, procurou-se, neste trabalho, estudar o efeito de pulverizações foliares de Paclobutrazol sobre a inibição da diferenciação floral natural do abacaxizeiro cv. Smooth Cayenne.

\section{MATERIAL E MÉTODOS}

O trabalho foi conduzido no período de maio de 2002 a fevereiro de 2004, no município de Presidente Alves-SP, latitude de $22^{\circ} 06^{\prime} 66^{\prime \prime}$ sul e longitude de $49^{\circ} 26^{\prime} 17^{\prime \prime}$ oeste e características climáticas conforme apresentadas na Tabela 1.

$\mathrm{O}$ experimento foi conduzido em solo Argilossolo Vermelho-Amarelo (PVA) eutrófico, textura arenosa/média, suave ondulado (Oliveira et al., 1999).

O plantio da área foi realizado em 17 e 18 de maio de 2002, sendo utilizado a cultivar Smooth Cayenne, predominante nesta região. As mudas foram do tipo filhote, com massa média de 300 $\mathrm{g}$, plantadas em fileiras duplas, no espaçamento de $1,00 \times 0,50 \mathrm{x}$ $0,30 \mathrm{~m}$. A condução da cultura foi efetuada conforme recomendações do Boletim 100 (Raij et al., 1997) e Sampaio (1995).

Os tratamentos consistiram nas combinações de três concentrações $\left(100 ; 150\right.$ e $\left.200 \mathrm{mg} \mathrm{L}^{-1}\right)$ do produto conhecido comercialmente por Paclobutrazol ( $10 \%$ de i.a.) e três freqüências de aplicação (2; 3 e 4 vezes), conforme descrito na Tabela 2 . As pulverizações foram efetuadas nas primeiras horas do dia, com pulverizador costal manual S-10, com capacidade de 10 litros, com jato dirigido para a roseta foliar central $\left(50 \mathrm{~mL} \mathrm{planta}^{-1}\right)$. Aos tratamentos adicionou-se o surfatante não-iônico, alquil-fenolpoliglico-éter (Extravon) a $0,05 \%$.

O delineamento experimental empregado foi o de blocos casualizados, num total de 10 tratamentos e três repetições. As parcelas experimentais constaram de 40 plantas.

As avaliações referentes à inibição da floração foram realizadas semanalmente, a partir de 10-07-03, quando, nas plantas do tratamento-controle, as inflorescências começaram a aparecer na roseta floral, estendendo-se até 29-08-03, quando todas as plantas-controle apresentavam inflorescência visível no centro da roseta foliar, totalizando oito avaliações. A avaliação foi efetuada através da contagem das plantas com inflorescência visível no centro da roseta foliar.

A primeira aplicação dos tratamentos ocorreu na primeira quinzena do mês de abril (01-04-03), época definida em função do nível de crescimento vegetativo das plantas, baseado na massa fresca e comprimento da folha ' $D$ '. A massa fresca e o comprimento da folha ' $\mathrm{D}$ ' foram determinados no dia 26-03-03 (antes da primeira aplicação dos tratamentos) e 10-07-03 (após todas as aplicações dos tratamentos), a fim de observar-se o estádio de desenvolvimento vegetativo das plantas nas diferentes parcelas. Foram coletadas duas folhas ao acaso, nas linhas internas de cada parcela, acondicionadas em sacos plásticos umedecidos com água e submetida à pesagem individual em balança digital, com precisão de $0,01 \mathrm{~g}$, no Laboratório de Fisiologia Vegetal da UNESP - Bauru.

Para a definição dos tratamentos, como concentrações do produto, épocas e intervalo de aplicação, foi realizado estudo preliminar nos anos de 2001-2003, nas condições de Avaí-SP, onde se definiram as melhores concentrações do produto, e verificou-se que a aplicação iniciada em maio foi tardia, assim como o intervalo de aplicação de 30 dias foi ineficiente.

Em 15-08-03, realizou-se a indução floral das plantas mediante a pulverização foliar de 2-cloro-etil-fosfônico ou ethephon, conhecido comercialmente como "Ethrel", com $240 \mathrm{~g}$ $\mathrm{L}^{-1}$ de ethephon ( $200 \mathrm{~mL}$ de Ethrel $100 \mathrm{~L}^{-1}$ de água $+2 \mathrm{~kg}$ de uréia), em todas as plantas que não apresentavam a inflorescência visível na roseta foliar.

Após a aplicação dos tratamentos, foram realizadas observações visuais das plantas e dos frutos para verificar a ocorrência de anomalias induzidas pela ação dos reguladores vegetais.

Os dados obtidos foram submetidos à análise de regressão (dados de floração) e análise de variância (teste F), com comparação de médias pelo teste Tukey, a 5\% de probabilidade (dados de crescimento vegetativo).

\section{RESULTADOS E DISCUSSÃO}

A avaliação da massa fresca e do comprimento da folha 'D' (Tabela 3 ) evidenciou que, em geral, as plantas apresentaram níveis de crescimento vegetativo similares nas diversas parcelas experimentais antes da aplicação dos tratamentos.

Os valores de massa fresca da folha ' $\mathrm{D}$ ' $(69,40$ a 78,57 g) obtidos foram similares aos encontrados por Sampaio (1995), que, para a mesma época de plantio e a mesma cultivar, registrou valor médio de 62,37 g. De acordo com Silvy (1961), citado por Py \& Tisseau (1969), estima-se que, na Costa do Marfim, para a obtenção de frutos de $1,5 \mathrm{~kg}$, há necessidade de que a massa fresca das folhas ' $\mathrm{D}$ ' seja de aproximadamente $65 \mathrm{~g}$ no momento da indução floral. Barbosa (1997), utilizando a cv. Pérola no período de maio, antes de utilizar reguladores vegetais para a inibição da floração, obteve massa fresca média das folhas ' $D$ ' em torno de 63,3 g. Sampaio et al. (1998) consideraram boa a uniformidade de crescimento vegetativo em experimento com a cv. Smooth Cayenne, cujas folhas ' $D$ ' apresentassem massa fresca de $50 \mathrm{~g}$ no mês de maio, quando receberam a primeira aplicação dos tratamentos.

No mês de julho (Tabela 3), após a aplicação dos tratamentos e quando foi efetuada a primeira contagem das inflorescências acumuladas, uma nova avaliação das folhas ' $D$ ' foi feita para determinar se os tratamentos inibiram o crescimento das plantas. Isto não foi observado, pois não houve efeito significativo dos tratamentos com Paclobutrazol sobre o crescimento da folha ' $\mathrm{D}$ ', cuja massa fresca variou de 77,83 a $87,10 \mathrm{~g}$ nos diversos tratamentos. 
Quanto ao comprimento das folhas 'D' (Tabela 3), essas apresentavam comprimentos adequados para receberem os tratamentos $(82,67$ a $89,67 \mathrm{~cm})$, superando os valores obtidos por Barbosa (1997), de aproximadamente $80 \mathrm{~cm}$ para a cv. Pérola.

Quanto ao processo de diferenciação floral, Cunha et al. (2003) concluíram que o Paclobutrazol, quando aplicado em abril e maio, foi capaz de inibir e diminuir a iniciação floral do abacaxi cv. Pérola ( 2 e 3 aplicações à concentração de $240 \mathrm{mg} \mathrm{L}^{-1}$ ), e sugeriram a realização de trabalhos sob condições ambientais diferentes, principalmente com plantas da cv. Smooth Cayenne. Barbosa (1997) também recomendou novos estudos com reguladores vegetais a partir de abril, testando-se doses econômicas, em diversas localidades, diferentes épocas de aplicação e concentrações do produto, bem como utilização de cultivares locais.

Através dos resultados obtidos no trabalho, observouse que as concentrações de Paclobutrazol aplicadas em diferentes épocas (Tabela 2) inibiram a diferenciação floral (Tabelas 4 e 5).

Na Tabela 4, verifica-se que a testemunha (controle) apresentou, na terceira semana de avaliação (25-07-03), ou seja, aos 115 dias após a primeira aplicação dos tratamentos, mais de $50 \%$ das plantas com inflorescência aparente no centro da roseta foliar, indicando que ocorreu a diferenciação natural nos meses de maio e junho, pois, segundo Matos \& Sanches (1989), da diferenciação floral ao aparecimento da inflorescência, são necessários 42 dias. Neste período (maio e junho), houve decréscimo das temperaturas mínimas, média e máxima em relação ao mês anterior (Tabela 1), uma vez que a diferenciação floral natural está intimamente relacionada ao encurtamento do dia, bem como da diminuição das temperaturas, principalmente a noturna, e a baixa insolação (Reinhardt et al., 1986).

Ainda na Tabela 4, pode-se observar que, em relação à testemunha, todos os tratamentos apresentaram efeito de inibição da indução floral natural, principalmente sob a maior concentração (200 mg L $\mathrm{m}^{-1}$ ) e as maiores freqüências de aplicação (3 e 4 vezes), concordando com os resultados obtidos por Cunha et al. (2003) com a cv. Pérola, que obtiveram efeito de inibição na indução da diferenciação floral natural com ácido 2-(3-clorofenoxi) propiônico (ACP) e Paclobutrazol, nas concentrações de 90 e $240 \mathrm{mg} \mathrm{L}^{-1}$, respectivamente, aplicados duas ou três vezes durante os meses de abril e maio.

Aos 115 dias após a primeira aplicação dos tratamentos (25-07-03), o controle apresentava mais da metade das plantas com inflorescência visível no centro da roseta, enquanto, nos demais tratamentos, a porcentagem de inibição floral variou de 90,8 a 100\% (Tabela 4). Aos 150 dias após a aplicação dos tratamentos (29-08-03), o controle já apresentava 100\% de suas plantas com a inflorescência visível, enquanto os tratamentos com Paclobutrazol a $200 \mathrm{mg} \mathrm{L}^{-1}$, aplicados 3 e 4 vezes, apresentavam $100 \%$ de inibição floral natural. Neste tratamento, ocorreram, aos 158 dias após a primeira aplicação do regulador vegetal, alterações morfológicas caracterizadas pelo fechamento da roseta foliar, devido ao enrolamento e torção das folhas jovens no centro. Essas alterações não causaram danos severos às plantas que se recuperaram e produziram frutos normais. Barbosa et al. (1998), em estudo com abacaxi 'Pérola', com uso do
Paclobutrazol, obtiveram melhores resultados, inibição de 82,22\%, da concentração de $100 \mathrm{mg} \mathrm{L}^{-1}$, em 3 aplicações a intervalo de 15 dias, em junho, e Scott (1993) reduziu a floração precoce do abacaxizeiro com aplicações foliares de paclobutrazol de 55,2\% para $28,5 \%$, uma vez que, essa taxa era de aproximadamente 50 a $70 \%$ no verão.

Pelos resultados deste trabalho, verifica-se que 3 e 4 aplicações de Paclobutrazol nas concentrações de 150 e $200 \mathrm{mg}$ $\mathrm{L}^{-1}$,apresentaram porcentagens superiores a $90 \%$ de inibição da diferenciação floral. Isso ocorre, provavelmente, porque o paclobutrazol inibe a produção de etileno, sendo a diferenciação floral do abacaxi uma resposta fisiológica à elevação do teor de etileno no meristema apical (Burg \& Burg, 1966). A alta concentração de ACC oxidase, em abacaxizeiro no estádio reprodutivo, pode indicar que o etileno seja um dos fatores envolvidos na indução floral. Min \& Bartholomew (1996), em estudo com abacaxizeiro, relataram que a produção de ACC não foi afetada pelo uso de paclobutrazol, enquanto a síntese de ACC oxidase, que converte ACC para etileno, foi inibida. Houve atraso significativo no aparecimento da inflorescência em resposta a todas as concentrações testadas, com maior atraso nas concentrações maiores.

A ação do Paclobutrazol na inibição da síntese de etileno é descrita por vários autores, sugerindo que a conversão de ACC a etileno, catalisada pela ACC oxidase, é influenciada pelos inibidores vegetais (Wang \& Steffens, 1985; Tari \& Mihalik, 1998). Sauerbrey et al. (1988) afirmaram que os inibidores vegetais podem provocar a inibição da síntese do etileno, pois, em plântulas de girassol, a formação de etileno foi reduzida significativamente por triazóis. A inibição foi acompanhada pela elevação no nível de ACC (1-aminociclopropano-1-carboxílico), enquanto os níveis de N-malonil-ACC permaneceram inalterados, sugerindo que a conversão de ACC a etileno, catalisada pela enzima ACC oxidase, foi influenciada pelos inibidores vegetais.

No presente trabalho, realizado com a cv. Smooth Cayenne e em região com condições climáticas diferentes, não houve redução significativa no tamanho da folha ' $\mathrm{D}$ ' após a aplicação dos tratamentos em relação ao controle (Tabela 3). Portanto, neste caso, não houve relação entre inibição da floração e redução do porte vegetativo da planta, como sugeriram Cunha et al. (2003). Além da clima e da cultivar, outro fator que pode ter determinado a divergência entre os resultados, nos dois trabalhos, é a diferença no porte das plantas no momento da aplicação do regulador, sendo que um porte vegetativo maior normalmente torna menos acentuado o efeito inibidor do regulador sobre o crescimento vegetativo da planta (Rebolledo et al., 1997). 
TABELA 1 -Precipitação mensal $(\mathrm{mm})$ e temperaturas máximas, mínimas e médias mensais $\left({ }^{\circ} \mathrm{C}\right)$ da região de Bauru-SP. Instituto de Pesquisas Meteorológicas (IPMET) da UNESP, Bauru, 2003/2004.

\begin{tabular}{|c|c|c|c|c|c|c|c|c|}
\hline \multirow[b]{3}{*}{ Meses } & \multicolumn{2}{|c|}{$\begin{array}{l}\text { Precipitação } \\
(\mathrm{mm})\end{array}$} & \multicolumn{2}{|c|}{ T.Máx. $\left({ }^{\circ} \mathrm{C}\right)$} & \multicolumn{2}{|c|}{ T. Média $\left({ }^{\circ} \mathrm{C}\right)$} & \multicolumn{2}{|c|}{ T.Min. $\left({ }^{\circ} \mathrm{C}\right)$} \\
\hline & & & & Ano & & & & \\
\hline & 2003 & 2004 & 2003 & 2004 & 2003 & 2004 & 2003 & 2004 \\
\hline Janeiro & 417,3 & 265,3 & 28,8 & 28,9 & 24,4 & 26,5 & 20,4 & 19,3 \\
\hline Fevereiro & 160,8 & 162,3 & 31,3 & 29,3 & 24,9 & 27,0 & 20,8 & 18,9 \\
\hline Março & 95,1 & - & 29,2 & - & 24,6 & - & 19,5 & - \\
\hline Abril & 173,6 & - & 28,0 & - & 23,9 & - & 17,7 & - \\
\hline Maio & 3,4 & - & 27,4 & - & 20,4 & - & 13,9 & - \\
\hline Junho & 56,0 & - & 27,0 & - & 19,3 & - & 15,3 & - \\
\hline Julho & 11,9 & - & 26,2 & - & 16,3 & - & 13,9 & - \\
\hline Agosto & 29,5 & - & 25,8 & - & 16,8 & - & 12,6 & - \\
\hline Setembro & 16,6 & - & 29,3 & - & 17,9 & - & 15,1 & - \\
\hline Outubro & 95,2 & - & 30,0 & - & 20,2 & - & 16,6 & - \\
\hline Novembro & 143,7 & - & 29,6 & - & 19,5 & - & 17,9 & - \\
\hline Dezembro & 273,7 & - & 30,3 & - & 20,3 & - & 19,6 & - \\
\hline
\end{tabular}

TABELA 2 - Tratamentos e épocas de aplicação do Paclobutrazol (2003).

\begin{tabular}{lcccc}
\hline Tratamentos & $\begin{array}{c}\text { ABRIL } \\
1^{\mathrm{a}} \\
\text { quinzena } \\
\text { Dosagens } \\
\text { de PBZ* } \\
\mathrm{mg} \mathrm{L}^{-1}\end{array}$ & $\begin{array}{c}\text { ABRIL } \\
2^{\mathrm{a}} \\
\text { quinzena } \\
\text { Dosagens } \\
\text { de PBZ* } \\
\mathrm{mg} \mathrm{L}^{-1}\end{array}$ & $\begin{array}{c}\text { MAIO } \\
1^{\mathrm{a}} \\
\text { quinzena } \\
\text { Dosagens } \\
\text { de PBZ* } \\
\mathrm{mg} \mathrm{L}^{-1}\end{array}$ & $\begin{array}{c}\text { MAIO } \\
2^{\mathrm{a}} \\
\text { quinzena } \\
\text { Dosagens } \\
\text { de PBZ* } \\
\mathrm{mg} \mathrm{L}^{-1}\end{array}$ \\
\hline $1-100 \mathrm{mg} \mathrm{L}^{-1}(4 \mathrm{x})$ & 100 & 100 & 100 & 100 \\
$2-150 \mathrm{mg} \mathrm{L}^{-1}(4 \mathrm{x})$ & 150 & 150 & 150 & 150 \\
$3-100 \mathrm{mg} \mathrm{L}^{-1}(3 \mathrm{x})$ & 100 & 100 & 100 & - \\
$4-150 \mathrm{mg} \mathrm{L}^{-1}(3 \mathrm{x})$ & 150 & 150 & 150 & - \\
$5-100 \mathrm{mg} \mathrm{L}^{-1}(2 \mathrm{x})$ & - & 100 & 100 & - \\
$6-150 \mathrm{mg} \mathrm{L}^{-1}(2 \mathrm{x})$ & - & 150 & 150 & - \\
$7-200 \mathrm{mg} \mathrm{L}^{-1}(4 \mathrm{x})$ & 200 & 200 & 200 & 200 \\
$8-200 \mathrm{mg} \mathrm{L}^{-1}(3 \mathrm{x})$ & 200 & 200 & 200 & - \\
$9-200 \mathrm{mg} \mathrm{L}^{-1}(2 \mathrm{x})$ & - & 200 & 200 & - \\
$10($ controle $)$ & água & água & água & água \\
\hline
\end{tabular}

* PBZ- Paclobutrazol
TABELA 3 - Massa fresca (g) e comprimento (cm) da folha ' $D$ ' do abacaxizeiro 'Smooth Cayenne' antes e depois dos tratamentos com Paclobutrazol. Presidente Alves (SP), 2004.

\begin{tabular}{|c|c|c|c|c|}
\hline \multirow[t]{2}{*}{ Tratamentos } & \multicolumn{2}{|c|}{$\begin{array}{c}\text { Massa fresca folha ' } \mathrm{D} \text { ' } \\
(\mathrm{g})\end{array}$} & \multicolumn{2}{|c|}{$\begin{array}{l}\text { Comprimento folha'D' } \\
(\mathrm{cm})\end{array}$} \\
\hline & $26 / 03 / 2003$ & $10 / 07 / 03$ & $26 / 03 / 2003$ & $10 / 07 / 03$ \\
\hline $1-100 \mathrm{mg} \mathrm{L}^{-1}(4 \mathrm{x})$ & $73,37 \mathrm{a}$ & $84,33 \mathrm{a}$ & $86,33 \mathrm{a}$ & $86,67 \mathrm{a}$ \\
\hline $2-150 \mathrm{mg} \mathrm{L}^{-1}(4 \mathrm{x})$ & $69,40 \mathrm{a}$ & $79,83 \mathrm{a}$ & $84,33 \mathrm{a}$ & $86,00 \mathrm{a}$ \\
\hline $3-100 \mathrm{mg} \mathrm{L}^{-1}(3 \mathrm{x})$ & $73,73 \mathrm{a}$ & $82,30 \mathrm{a}$ & $86,00 \mathrm{a}$ & $87,00 \mathrm{a}$ \\
\hline 4- $150 \mathrm{mg} \mathrm{L}^{-1}(3 \mathrm{x})$ & $73,90 \mathrm{a}$ & 79,83 a & $85,33 \mathrm{a}$ & 87,67 a \\
\hline $5-100 \mathrm{mg} \mathrm{L}^{-1}(2 \mathrm{x})$ & $75,10 \mathrm{a}$ & $82,27 \mathrm{a}$ & $89,67 \mathrm{a}$ & 89,00 a \\
\hline $6-150 \mathrm{mg} \mathrm{L}^{-1}(2 \mathrm{x})$ & $71,97 \mathrm{a}$ & $77,83 \mathrm{a}$ & $87,67 \mathrm{a}$ & $87,00 \mathrm{a}$ \\
\hline $7-200 \mathrm{mg} \mathrm{L}^{-1}(4 \mathrm{x})$ & $69,90 \mathrm{a}$ & 79,33 a & 85,67 a & 89,67 a \\
\hline $8-200 \mathrm{mg} \mathrm{L}^{-1}(3 \mathrm{x})$ & $72,97 \mathrm{a}$ & $86,07 \mathrm{a}$ & $82,67 \mathrm{a}$ & 88,33 a \\
\hline $9-200 \mathrm{mg} \mathrm{L}^{-1}(2 \mathrm{x})$ & $75,27 \mathrm{a}$ & $81,97 \mathrm{a}$ & $86,00 \mathrm{a}$ & $92,33 \mathrm{a}$ \\
\hline 10 (controle) & $78,57 \mathrm{a}$ & $87,10 \mathrm{a}$ & $87,33 \mathrm{a}$ & $88,67 \mathrm{a}$ \\
\hline C.V. $(\%)$ & 6,35 & 8,21 & 5,65 & 5,12 \\
\hline
\end{tabular}

Médias seguidas de mesma letra não diferem significativamente entre si, pelo teste Tukey, a $5 \%$ de probabilidade.

TABELA 4- Porcentagem de abacaxizeiros cv. Smooth Cayenne com diferenciação floral inibida nos diferentes tratamentos estudados. Presidente Alves (SP), 2004.

\begin{tabular}{|c|c|c|c|c|c|c|c|c|c|}
\hline \multirow[b]{2}{*}{ Tratamentos } & \multicolumn{7}{|c|}{ Avaliações } & \multirow[b]{2}{*}{$22-08-03$} & \multirow[b]{2}{*}{$29-08-03$} \\
\hline & $10-07-03$ & & $16-07-03$ & $25-07-03$ & $31-07-03$ & $08-08-03$ & $15-08-03$ & & \\
\hline \multicolumn{10}{|c|}{$\%$ de plantas } \\
\hline $1-100 \mathrm{mg} \mathrm{L}^{-1}(4 \mathrm{x})^{3}$ & 100,0 & 100,0 & & 98,3 & 97,5 & 95,0 & 95,0 & 95,0 & 95,0 \\
\hline $2-150 \mathrm{mg} \mathrm{L}^{-1}(4 \mathrm{x})$ & 100,0 & 100,0 & & 100,0 & 100,0 & 98,3 & 98,3 & 98,3 & 98,3 \\
\hline $3-100 \mathrm{mg} \mathrm{L}^{-1}(3 \mathrm{x})^{2}$ & 98,3 & 98,3 & & 98,3 & 96,7 & 93,3 & 93,3 & 93,3 & 86,7 \\
\hline $4-150 \mathrm{mg} \mathrm{L}^{-1}(3 \mathrm{x})$ & 98,3 & 98,3 & & 98,3 & 97,5 & 95,8 & 95,8 & 95,8 & 94,2 \\
\hline $5-100 \mathrm{mg} \mathrm{L}^{-1}(2 \mathrm{x})^{1}$ & 100,0 & 100,0 & & 93,3 & 86,7 & 83,3 & 83,3 & 83,3 & 77,5 \\
\hline $6-150 \mathrm{mg} \mathrm{L}^{-1}(2 \mathrm{x})$ & 98,3 & 94,2 & & 91,7 & 89,2 & 88,3 & 88,3 & 88,3 & 88,3 \\
\hline $7-200 \mathrm{mg} \mathrm{L}^{-1}(4 \mathrm{x})$ & 100,0 & 100,0 & & 100,0 & 100,0 & 100,0 & 100,0 & 100,0 & 100,0 \\
\hline $8-200 \mathrm{mg} \mathrm{L}^{-1}(3 \mathrm{x})$ & 100,0 & 100,0 & & 100,0 & 100,0 & 100,0 & 100,0 & 100,0 & 100,0 \\
\hline $9-200 \mathrm{mg} \mathrm{L}^{-1}(2 \mathrm{x})$ & 94,2 & 93,3 & & 90,8 & 89,2 & 89,2 & 89,2 & 89,2 & 86,7 \\
\hline $10($ controle $)$ & 97,5 & 96,7 & & 47,5 & 32,5 & 23,3 & 23,3 & 23,3 & 0,0 \\
\hline
\end{tabular}

${ }^{1)}$ Aplicação do Paclobutrazol na segunda quinzena de abril e primeira quinzena de maio de 2003 .

(2) Aplicação do Paclobutrazol na primeira e segunda quinzenas de abril e primeira quinzena de maio de 2003.

(3) Aplicação do Paclobutrazol na primeira e segunda quinzenas de abril, primeira e segunda quinzenas de maio de 2003. 
TABELA 5- Equações de regressão, representativas da evolução de emissão de inflorescências no centro da roseta foliar, em plantas de abacaxi 'Smooth Cayenne', nos diferentes tratamentos com Paclobutrazol. Presidente Alves (SP), 2004.

\begin{tabular}{|c|c|c|}
\hline Tratamentos & Modelo ajustado ${ }^{1}$ & $* \mathrm{R}^{2}(\%)$ \\
\hline $1-100 \mathrm{mg} \mathrm{L}^{-1}(4 \mathrm{x})$ & $Y=-0,2143+0,3254 x$ & 87,96 \\
\hline $2-150 \mathrm{mg} \mathrm{L}^{-1}(4 \mathrm{x})$ & $\mathrm{Y}=0,33$ & , \\
\hline $3-100 \mathrm{mg} \mathrm{L}^{-1}(3 \mathrm{x})$ & $Y=1,7143-1,3838 x+0,4322 x^{2}-0,0253 x^{3}$ & 99,30 \\
\hline 4- $150 \mathrm{mg} \mathrm{L}^{-1}(3 \mathrm{x})$ & $Y=0,250+0,250 x$ & 94,90 \\
\hline $5-100 \mathrm{mg} \mathrm{L}^{-1}(2 \mathrm{x})$ & $Y=-1,4048+1,4048 x$ & 94,96 \\
\hline $6-150 \mathrm{mg} \mathrm{L}^{-1}(2 \mathrm{x})$ & $Y=-0,1429+1,5397 x-0,119 x^{2}$ & 98,87 \\
\hline $7-200 \mathrm{mg} \mathrm{L}^{-1}(4 \mathrm{x})$ & $\mathrm{Y}=0$ & - \\
\hline $8-200 \mathrm{mg} \mathrm{L}^{-1}(3 \mathrm{x})$ & $\mathrm{Y}=0$ & \\
\hline $9-200 \mathrm{mg} \mathrm{L}^{-1}(2 \mathrm{x})$ & $\mathrm{Y}=2,1429+0,4127 \mathrm{x}$ & 91,97 \\
\hline 10 (controle) & $Y=-13,6191+12,5992 x-0,7579 x^{2}$ & 88,52 \\
\hline
\end{tabular}

* $\mathrm{P}<0,001$ para todos os modelos ajustados. ${ }^{1} \mathbf{Y}=\mathbf{a}+\mathbf{b}_{1} \mathbf{x}+\mathbf{b}_{2} \mathbf{x}^{2}+\mathbf{b}_{3} \mathbf{x}^{3}$

\section{CONCLUSÕES}

Nas condições deste experimento conduzido na região do Centro-Oeste Paulista, pode-se concluir que:

1-O controle da diferencição floral natural do abacaxizeiro 'Smooth Cayenne' com Paclobutrazol deve ser iniciado em abril, usando-se intervalos de aplicações de 15 dias.

2-O Paclobutrazol inibiu o processo de diferenciação floral natural do abacaxizeiro 'Smooth Cayenne' em todos os tratamentos estudados, recomendando-se o seu uso na concentração de $150 \mathrm{mg} \mathrm{L}^{-1}$, em duas pulverizações foliares.

\section{REFERÊNCIAS}

BARBOSA, N.M.L. Efeito de reguladores e da adubação nitrogenada no controle do florescimento natural precoce do abacaxizeiro. 1997. 59 f. Dissertação (Mestrado)- Universidade Federal da Bahia, Cruz das Almas, 1997.

BARBOSA, N.M.L.; CUNHA, G.A.P.; REINHARDT, D.H. Controle da floração natural do abacaxizeiro "Pérola" com uréia e reguladores de crescimento no Recôncavo Baiano. Revista Brasileira de Fruticultura, Jaboticabal, v.20, n.3, p.359-366, 1998.

BARBOSA, N.M.L.; CUNHA, G.A.P; REINHARDT, D.H.; BARROS, P.G.; SANTOS, A.R.L. Indução de alterações morfológicas e anatômicas em folhas de abacaxizeiro 'Pérola' pelo ácido 2-(3-clorofenoxi) propiônico. Revista Brasileira deFruticultura, Jaboticabal, v.25, n.3, p.386-389, 2003.

BURG, S.P.; BURG, E.A. Auxin-induced ethylene formation and its relation to flowering in the pineapple. Science, Washington, v.152, p.1269, 1966.

CUNHA, G.A. P.; COSTA, J.T.A.; REINHARDT, D.H. Natural flowering in pineapple: inhibition by growth regulators. Fruits, Paris, v.58, n.1, p.27-37, 2003.
FAO - Food and Agriculture Organization. FAOSTAT. Disponível em: $<$ http://www.fao.org.htm.>. Acesso em: 29 jul. 2004.

IBGE- INSTITUTO BRASILEIRO DE GEOGRAFIA E ESTATISTICA. Levantamento sistemático da produção agrícola. Brasília, 2004. Disponível em: $<\mathrm{http} / / /$ www.sidra.ibge.gov.br/ htm. >. Acesso em: 22 jul. 2004.

MATOS, A.P.; SANCHES, N.F. Desenvolvimento da inflorescência do abacaxizeiro 'Pérola'. Revista Brasileira de Fruticultura, Jaboticabal, v.11, n.2, p.49-53, 1989.

MIN, X.J.; BARTHOLOMEW, D.P. Effect of plant growth regulators on ethylene production, 1- aminocyclopropane-1carboxilic acid oxidase activity, and initiation of inflorescence development of pineapple. Journal of Plant Growth Regulation, New York, v.15, n.3, p.121-128, 1996.

OLIVEIRA, J.B.; CAMARGO, N.C.; ROSSI, M;FILHO, B.C. Mapa pedológico do Estado de São Paulo. Campinas: IAC, 1999. 64p.

PY, C.; TISSEAU, M. A. La pina tropical. Barcelona: Blume,1969. $278 \mathrm{p}$.

RABIE, E.C.; TUSTIN, H.A.; WESSON, K.T. Inhibition of natural flowering occurring during the winter months in queen pineapple in Kwazulu Natal, South Africa. Acta Horticulturae, Wageningen, v.529, p.175-183, 2000.

RAIJ, B.V.; CANTARELLA, H.; QUAGGIO, J.A. Recomendações de adubação e calagem para o Estado de São Paulo. Campinas: IAC, 1997.285p.

REBOLLEDO, A.M.; URIZA, D.E.A.; AGUIRRE, L.A.G.; PAPALOAN, C.E. Inhibicion de la floración de la piña con diferentes dosis de Fruitone CPA en dos densidades de siembra. Acta Horticulturae, Wageningen, v.495, p.247-354, 1997.

REINHARDT, D.H.R.C.; COSTA, J.T.A.; CUNHA, G.A.P. Influência da época de plantio, tamanho da muda e idade da planta para a indução floral do abacaxi 'Smooth Cayenne' no Recôncavo Baiano. I. Crescimento vegetativo, produção de mudas e florescimento natural. Fruits, Paris, v.41, n.1, p.31-41, 1986.

REINHARDT, D.H.R.C.; SOUZA, J.S. The pineapple industry in Brazil. Acta Horticulturae, Wageningen, v.529, p.57-71, 2000.

SAMPAIO, A. C.; FUMIS T.F.; HERNANDES, V.A.N. Ácido alfanaftalenoacético (ANA) no controle da diferenciação floral natural do abacaxizeiro cv. Smooth Cayenne. Revista Brasileira de Fruticultura, Jaboticabal, v.20, n.3, p.353-358, 1998.

SAMPAIO, A.C. Ação do nitrogênio, de épocas de plantio e do ácido 2,3-clorofenoxipropiônico sobre a produção do abacaxizeiro e características físico-químicas do fruto. 1995. 100 f. Tese 
(Doutorado) - Faculdade de Ciências Agrárias, Universidade Estadual Paulista, Botucatu, 1995.

SAMPAIO, A.C. Chat sobre abacaxicultura na região de Bauru. 2003. Disponível em:<http://www.todafruta.com.br/htm.>. Acesso em: 13 jan. 2004.

SAUERBREY,E.; GROSSMANN, K.; JUNG,J. Ethylene production by sunflower cell suspensions. Effects of plant growth retardants. Plant Physiology, Limburgerhof, v.87, p.510-513, 1988.

SCOTT, C.H. The effect of two plant growth regulators on the inhibition of precocious fruiting in pineapple. Acta Horticulturae, Wageningen, v.334, p.77-82,1993.
TARI, I.; MIHALIK, E. Comparison of the effects of white light and growth retardant paclobutrazol on the ethylene production in bean hypocotyls. Plant Growth Regulation, New York, v.24, p.67-72, 1998.

WANG, S. Y.; STEFFENS, G. L. Effect of paclobutrazol on water stress-induced ethylene biosynthesis and polyamine accumulation in apple seedling leaves. Phytochemistry, New York, v.24, n.10, p.2185-2190, 1985. 